\title{
A NEW STATISTICAL MODEL FOR NON-RAYLEIGH AMPLITUDE SIGNALS
}

\author{
Torbjørn Eltoft \\ Department of Physics, \\ University of Tromsø, \\ 9037 Troms $ø$, Norway
}

\begin{abstract}
We introduce a new statistical distribution for modeling nonRayleigh amplitude statistics. We call the new model the Rician Inverse Gaussian (RiIG) distribution. The theoretical basis of the model is briefly presented, and we give an EM-type algorithm for estimating its parameters from data. Finally, we include some modeling examples, where we have tested the ability to fit to histograms of linear and log-compressed medical ultrasound data.
\end{abstract}

\section{INTRODUCTION}

Modeling non-Rayleigh amplitude statistics in coherent imaging systems has been a research area for almost three decades, and several interesting distributions have been proposed. The most common of these are the Rice-distribution, the K-distribution and the homodined K-distribution. Accurate statistical models for the amplitude statistics are important both with respect to characterization/classification of image regions [1], and with respect to speckle filtering [2].

In this paper we introduce a new model for modeling the amplitude statistics of coherent imagery, which is a compound distribution of a the Rician distribution and the Inverse Gaussian (IG) [3]. We have for this reason denoted this new distribution the Rician Inverse Gaussian (RilG) distribution. We give some details of the properties of this new model, describe how its parameters can be estimated, and test its ability to fit to histograms of segments of ultrasound images.

\section{REVIEW OF SPECKLE MODELS}

If the scattering medium is modeled as a collection of discrete scatterers, we may write the detected signal as

$$
s=X+j Y=A e^{-j \Phi}=\sum_{i=1}^{N} a_{i} e^{j \phi_{i}},
$$

where $N$ is the number of scatterers, $a_{i}$ is the amplitude and $\phi_{i}$ is the phase of the $i$ th scatterer.

$i$ : Now, if the scatterers' phases are random and independent of the amplitudes, and if the number $N$ is very large, the probability density function (pdf) of $A$, the amplitude of $s$, will be a Rayleigh distribution

$$
p_{A}(a)=\frac{a}{\sigma^{2}} e^{-\frac{a^{2}}{2 \sigma^{2}}},
$$

where $\sigma$ is the the standard deviation of the Gaussian distributed $I$ - and $Q$-components of $s$. This distribution represents the fully-developed speckle case, or the diffuse scattering signal.

ii: If a specular component $A_{\mathrm{c}}$ is added to (1), the pdf of the amplitude gets Rician, i.e.

$$
p_{A}(a)=\frac{a}{\sigma^{2}} e^{-\frac{a^{2}+A_{c}^{2}}{2 \sigma^{2}}} I_{0}\left(\frac{a A_{c}}{\sigma^{2}}\right)
$$

where $I_{0}$ is the modified Bessel function of first kind and zero order.

iii: The well-known $K$-distribution results, if we allow the variance of the Rayleigh-distribution to itself being a random variable, distributed according to a $\Gamma$-distribution. In this case, the amplitude of $s$ is a compound variable of a Rayleigh variable and a $\Gamma$-distributed variable. The pdf is explicitly given as

$$
p_{A}(a)=\frac{2 b}{\Gamma(v)}\left(\frac{a b}{2}\right)^{v} K_{v-1}(b a),
$$

where $K_{v}(\cdot)$ is the modified Bessel function of second kind and order $v, b=2 \sqrt{v /\left\langle a^{2}\right\rangle}$ is a scale parameter. $v$ is a shape parameter.

iv: The homodyned $\mathrm{K}$-distribution results when a coherent component, $A_{c}$, is added to the diffuse scattering signal in (1), and we allow the number of terms in the summation, $N$, to itself being random following a negative binomial distribution. The resulting pdf, which can not be obtained in closed form, is given by the integral

$$
p_{A}(a)=a \int_{0}^{\infty} \frac{u J_{0}\left(u A_{c}\right) J_{0}(u a)}{\left(1+u^{2} \sigma^{2} / 2 v\right)^{v}} d u .
$$

The models reviewed above have some shortcomings: the Rayleigh model certainly is an appropriate model for fullydeveloped speckle, but this case does not often occur in 
high-resolution imagery. Also, the K-model has its limitations, because, often the signal has a specular component, resulting in bad fits to the data. The homodyned model has good modeling capabilities, but its use is restricted by the lack of a tractable pdf.

\section{NEW MODEL}

We will in the following introduce a unifying model for non-Rayleigh amplitude data, which is able to overcome the problems mentioned above. In addition, its parameters can easily be estimated from data.

When the $I$ - and $Q$-components of the detected complex signal deviate from Gaussian statistics, the amplitude will be non-Rayleigh. We propose to use the Normal Inverse Gaussian (NIG) distribution to model these components. A NIG-distribution is defined as a variance-mean mixture of a normal distribution with the inverse Gaussian $(I G)$ as a mixing distribution [4]. The probability density function is explicitly given as

$$
p_{N I G}(x ; \alpha, \beta, \mu, \delta)=\frac{\alpha}{\pi} \frac{\exp (p(x))}{q(x)} K_{1}(\delta \alpha q(x)),
$$

where

$$
\begin{gathered}
p(x)=\delta \sqrt{\alpha^{2}-\beta^{2}}+\beta(x-\mu), \\
q(x)=\frac{1}{\delta} \sqrt{(x-\mu)^{2}+\delta^{2}},
\end{gathered}
$$

and $K_{1}(\cdot)$ is the modified Bessel function of the second kind and order 1. As noted, the distribution is characterized by the four parameters $(\alpha, \beta, \mu, \delta)$, which are bounded such that $0 \leq|\beta| \leq \alpha, \delta$, and $-\infty<\mu<\infty$. $\alpha$ controls the steepness of the distribution; a small $\alpha$ means a steep distribution, a larger $\alpha$ means a less steep distribution. $\beta$ determines the skewness; $\beta<0$ implies skewed to the left, $\beta>0$ implies skewed to the right, and $\beta=0$ implies a symmetric distribution. $\mu$ is a location parameter, and $\delta$ is a dispersion parameter similar to the variance in the Gaussian distribution.

Let $X$ be defined by the relation

$$
X=\mu+\beta Z+\sqrt{Z} N
$$

where $N \sim \mathcal{N}(0,1)$. Hence, the conditional distribution $X \mid Z$ is normal $\mathcal{N}(\mu+\beta z, z)$. The marginal distribution of $Z$ is Inverse Gaussian (IG) with parameters $\delta$ and $\gamma=\sqrt{\alpha^{2}-\beta^{2}}$. This probability density is given by

$$
p_{I G}(z ; \delta, \gamma)=\frac{\delta}{\sqrt{2 \pi}} \exp (\delta \gamma) z^{-3 / 2} \exp \left(-\frac{1}{2}\left(\frac{\delta^{2}}{z}+\gamma^{2} z\right)\right) \text {. }
$$

The IG-distribution was first introduced as the fi rst passage time distribution of Brownian motion with drift. It has recently attained increasing interest in signal processing and statistics (financial) due to its ability to model highly skewed densities, and its many analogies with the Gaussian distribution. Its mean and variance are $E\{Z\}=\frac{\delta}{\gamma}$ and $V\{Z\}=\frac{\delta}{\gamma^{3}}$, respectively.

Consider the case when the $X$ - and $Y$-components of $s$ in (1) are uncorrelated and 2-dimensional NIG-distributed, i.e: $X \sim N I G\left(0, \alpha, \beta_{x}, \delta\right)$, and $Y \sim N I G\left(0, \alpha, \beta_{y}, \delta\right)$. Note that we assume that both $\mu_{x}$ and $\mu_{y}$ are zero. Accordingly, $X$ and $Y$ are both compound random variables of the form: $X=\beta_{x} Z+\sqrt{Z} N_{x}$ and $Y=\beta_{y} Z+\sqrt{Z} N_{y}$, where $N=\left(N_{x}, N_{y}\right) \sim$ $\mathcal{N}(0, \Sigma)$, with $\Sigma=\tilde{I}$ ( $\tilde{I}$ is the 2-dimensional identity matrix.), and $Z \sim I G(\delta, \gamma)$, with $\gamma=\sqrt{\alpha^{2}-\left(\beta_{x}^{2}+\beta_{y}^{2}\right)}$. It is now easy to show that the distribution of the envelope, conditioned on $Z$, i.e. $A \mid Z$, is Rician distributed, with pdf given by

$$
p_{A \mid Z}(a \mid z)=\frac{a}{z} \exp \left(-\frac{1}{2 z}\left(a^{2}+\beta^{2} z^{2}\right)\right) I_{0}(\beta a)
$$

where $\beta=\sqrt{\beta_{x}^{2}+\beta_{y}^{2}}$. From (9) we observe that the skewness parameters of the NIG-distributions will have the effect of a coherent component on the amplitude statistics. Now, the amplitude will accordingly be a compound variable consisting of the Rice-distribution in $(9)$ and the $\mathrm{IG}(\delta, \gamma)$ distribution, i.e.

$$
p_{A}(a)=\int_{0}^{\infty} p_{A \mid Z}(a \mid z) p_{Z}(z) d z .
$$

The integral in (10) can be evaluated to give the following closed form pdf for $A$ :

$$
\begin{aligned}
p_{A}(a)=\sqrt{\frac{2}{\pi}} \alpha^{3 / 2} \delta \exp (\delta \gamma) & \\
& \cdot \frac{a}{\left(\delta^{2}+a^{2}\right)^{3 / 4}} K_{3 / 2}\left(\alpha \sqrt{\delta^{2}+a^{2}}\right) I_{0}(\beta a) .
\end{aligned}
$$

We call this distribution the Rician Inverse Gaussian (RiIG) distribution. $A$ s can be seen, the pdf has three parameter. Fig.1 displays some realizations of the pdf for some selected values of the parameters. The numbers in brackets in the upper right corner of Fig. 1 give the values of $\alpha, \beta$, and $\delta$ for each curve. We observe that by varying the parameters $\alpha$, $\boldsymbol{\beta}$, and $\delta$, we may model a wide range of distributions, from a Rayleigh-shaped pdf to a Gaussian-shaped pdf.

\section{PARAMETER ESTIMATION}

The parameters of the RiIG distribution can be estimated using an EM-type algorithm. In the previous section we assumed that the speckled free image, represented by the variable $z$, follows an $\operatorname{IG}(z ; \delta, \gamma)$ distribution with parameters $\delta$ and $\gamma$. This distribution is well documented in the literature [3]. Its $k$ th-order moments are given by

$$
m_{k}=E\left\{Z^{k}\right\}=\left(\frac{\delta}{\gamma}\right)^{k} \frac{K_{k-\frac{1}{2}}(\gamma \delta)}{K_{-\frac{1}{2}(\gamma \delta)}} .
$$




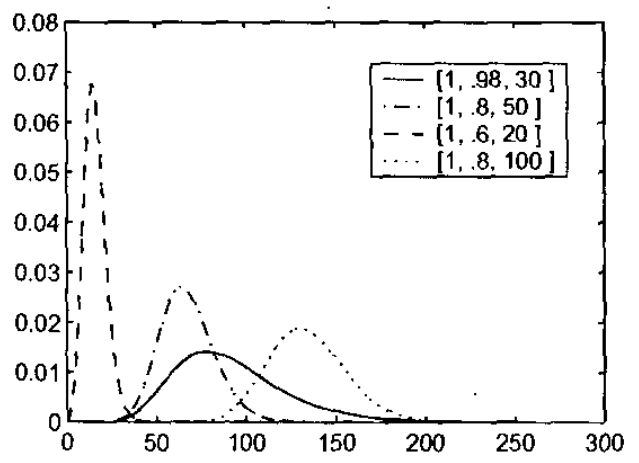

Fig. 1. Examples of pdfs residing to the RiIG model. The numbers in the brackets correspond to $\alpha, \beta, \delta$, respectively

From (12) we easily find that $E\{Z\}$ and $E\left\{\frac{1}{Z}\right\}$ are:

$$
\begin{gathered}
E\{Z\}=\frac{\delta}{\gamma} \\
E\left\{\frac{1}{Z}\right\}=\left(\frac{\delta}{\gamma}\right)^{-1}\left(\frac{1}{\gamma \delta}+1\right) .
\end{gathered}
$$

It is further noted that the posterior distribution $Z \mid A$ is given as:

$$
\begin{aligned}
p_{Z \mid A}(z \mid a)= & \frac{p_{A \mid Z}(a \mid z) p_{Z}(z)}{p_{A}(a)} \\
= & \left(\frac{\alpha}{\sqrt{\delta^{2}+a^{2}}}\right)^{-\frac{3}{2}} \frac{z^{\left(-\frac{3}{2}\right)-1}}{2 K_{-\frac{3}{2}}\left(\alpha \sqrt{\delta^{2}+a^{2}}\right)} \\
& \times \exp \left(-\frac{1}{2}\left(\frac{\delta^{2}+a^{2}}{z}+\alpha^{2} z\right)\right) .
\end{aligned}
$$

This is the pdf of a generalized inverse Gaussian (GIG) variable specified by the parameters $\left(-\frac{3}{2}, \sqrt{\delta^{2}+a^{2}}, \alpha\right)$ [4]. The moments of a $\operatorname{GIG}(z ; \lambda, \delta, \gamma)$ distribution are given by [4]

$$
E\left\{Z^{k}\right\}=\left(\frac{\delta}{\gamma}\right)^{k} \frac{K_{\lambda+k}(\delta \gamma)}{K_{\lambda}(\delta \gamma)} .
$$

Let $v=\sqrt{\delta^{2}+a^{2}}$. We thus have that

$$
\begin{gathered}
E\{Z \mid A\}=\frac{v}{\alpha} \frac{v \alpha}{1+v \alpha}, \text { and } \\
E\left\{\frac{1}{Z} \mid A\right\}=\frac{v}{\alpha} \frac{(v \alpha)^{2}+3 v \alpha+3}{v \alpha(1+v \alpha)} .
\end{gathered}
$$

Now, given $\mathrm{N}$ independent observations $a_{i}, i=1,2, \cdots, N$. From some initial values for $\alpha, \beta$, and $\delta$, we may estimate $E\{Z\}$ as

$$
<>=\frac{1}{N} \sum_{i=1}^{N}\left(\frac{v_{i}}{\alpha} \frac{v_{i} \alpha}{1+v_{i} \alpha}\right)
$$

and $E\left\{\frac{1}{2}\right\}$ as

$$
<\frac{1}{Z}>=\frac{1}{N} \sum_{i=1}^{N}\left(\frac{v_{i}}{\alpha} \frac{\left(v_{i} \alpha\right)^{2}+3 v_{i} \alpha+3}{v_{i} \alpha\left(1+v_{i} \alpha\right)}\right) .
$$

Using (13) and (14), our estimators for $\delta$ and $\gamma$ are

$$
\begin{array}{r}
\hat{\delta}=\sqrt{\left(\frac{1}{\frac{1}{<2\rangle}}-\frac{1}{<Z\rangle}\right),} \\
\hat{\gamma}=\frac{\hat{\delta}}{<Z\rangle},
\end{array}
$$

respectively. The $\beta$ parameter is estimated using the maximum likelihood method. Using previous estimates of $\alpha, \beta$, $\delta$, and $\gamma$, define $F(\beta ; a)$ as

$$
F(\beta ; a)=\log \prod_{i=1}^{N} p_{A}\left(a_{i}\right),
$$

where $p_{A}\left(a_{i}\right)$ is defined in (11). Our estimate for $\beta$ is accordingly given as

$$
\hat{\boldsymbol{\beta}}=\arg \max _{\beta} F(\beta ; a) .
$$

This maximization must be performed numerically.

\section{MODELING ULTRASOUND DATA}

Fig. 2 shows a log-compressed medical ultrasound image of the human heart. We have tested the modeling capability of the RilG-model on both the linear image (un-compressed), and the log-compressed version. No pre-processing has been done. In the left panels of Figs.3-5 the histograms of the amplitudes of the marked regions in Fig. 2 have been displayed, along with the best fitted RilG- and K-models. The parameters of the K-models were estimated using a moment based estimator, or by matching the moments of the data. In the right panels we have included variance stabilized p-p-plots of the corresponding models. For the log-compressed data the $\mathrm{K}$ pdf is not an appropriate model, and hence comparisons with this model have been skipped. We note that for the linear data the RiIG-model seems to fit the histograms of the data much better than the K-model. In Fig. 3 and Fig. 5 we see that the $\mathrm{K}$-model is not able to follow the shape of the histograms, whereas the RiIG model does. The RiIGmodel also provides excellent fits to the histograms of the log-compressed data.

\section{CONCLUSION}

We have presented a new statistical distribution for modeling amplitude data which do not follow a Rayleigh distribution. We call this distribution a Rician Inverse Gaussian 


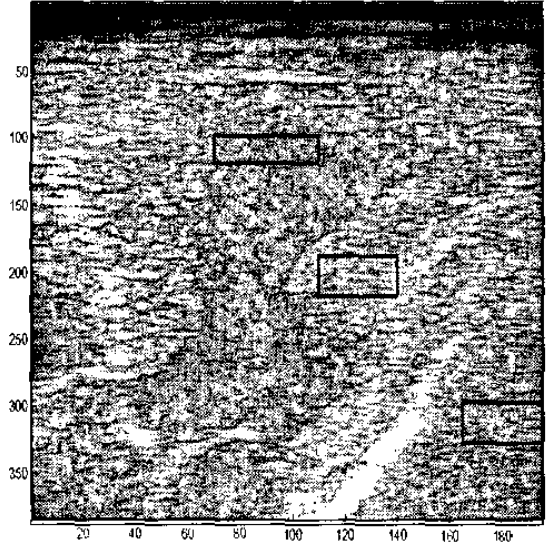

Fig. 2. Ultrasound image (log-compressed). The marked areas define the image regions which the RilG-model was tested on.
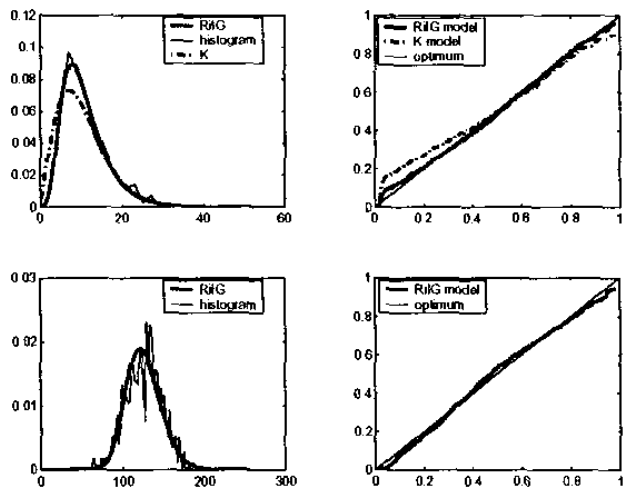

Fig. 3. Linear data. Fitted RilG-model (*), histogram (dashed) and fitted K-model (solid) corresponding to the four image regions in Fig.2.

distribution. The preliminary tests show that this model has a large degree of flexibility, and may be applied in situations where existing models, like the $\mathrm{K}$-distribution, fails.

\section{REFERENCES}

[1] R. M. Cramblitt and M. R. Bell, "Surface characterization using frequency diverse scattering measurements and regularity models," IEEE Trans. Signal Processing, vol. 44, no. 3, pp. 599-610, March 1996.

[2] S. Solbø and T. Eltoft, "Г-WMAP: A statistical speckle filter operating in the wavelet domain," Int. J. Remote Sens, 2003, accepted for publication.

[3] M. T. Wasan, "First passage time distribution of brown-
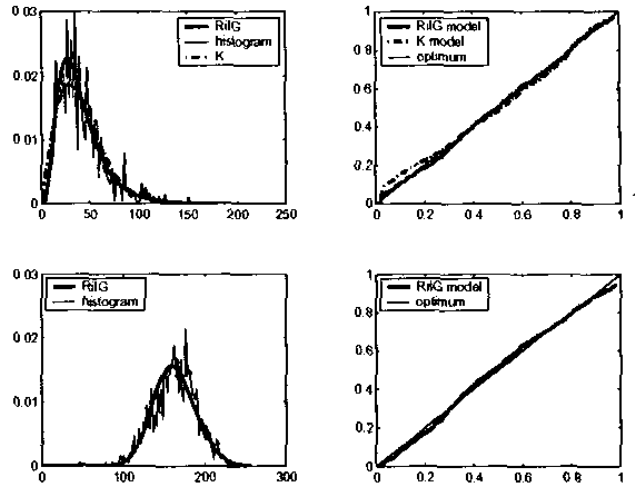

Fig. 4. Log-compressed data. Fitted RilG-model (solid) and histogram (dashed) corresponding to the four image regions in Fig.2.
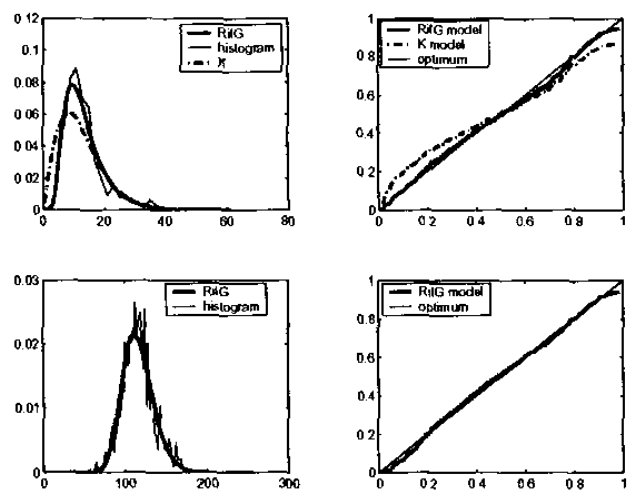

Fig. 5. Log-compressed data. Fitted RilG-model (solid) and histogram (dashed) corresponding to the four image regions in Fig. 2 .

ian motion with positive drift (inverse gaussian distribution)," Queen's papers in pure and applied matematics -no.19, Queen's University, 1969.

[4] O. E. Barndorff-Nielsen, "Normal Inverse Gaussian Distributions and Stochastic Volatility Modelling," Scand. J. Statist., vol. 24, pp. 1-13, 1997. 